\title{
A Photoelectric Investigation of the Eclipsing Binary V 78 in Omega Centauri (NGC 5139)
}

\author{
EDWARD H. GEYER (Bonn)
}

The existence of eclipsing binaries, though extremely rare, in stellar systems like globular clusters, should attract the observers, because one could obtain fundamental data on highly evolved stars. The variable star V 78 in the globular cluster NGC 5139, which was found by BAILEY (1901), is one of these rare cases. MARTIN (1938) derived the period P $=1$ 168118, and showed by means of an extensive photographic photometry that it is an Algol type eclipsing binary. Recently SISTERO et al. $(1968,1969)$ rediscussed the observations of MARTIN, and with additional measurements derived the following light elements:

$$
\text { Min }=\mathrm{JD}_{\mathrm{hel}} 2430066,9693+1 \mathrm{~d} 1681288 \cdot \mathrm{E} .
$$

They also found indications for a shallow secondary minimum, and presented a preliminary solution for the double star's elements.

Based on the Greenwich proper motion investigations of the cluster DICKENS and SAUNDERS showed in 1965 that V 78 is not a foreground star, but most likely a cluster member. Short afterwards I (GEYER, 1967) published a colour-magnitude diagram of Omega Centauri, and from numerous photographic observations in two colours of V 78 I could place its position in the HR diagram, where it lies slightly above the RR Lyrae gap of the horizontal branch of the $c-m$ diagram of the cluster. This fact attracted my attention for further observations of this object, because it seems feasible to derive observationally for the first time the dimensions of a horizontal branch star.

During a $2^{1 / 2}$ months stay at the ESO Observatory at La Silla mountain/Chile in May to July $1968 \mathrm{I}$ observed V 78 on 15 nights of photometric quality with the standard photoelectric equipment of the $1 \mathrm{~m}$ Cassegrain telescope in UBV. Some 200 measurements were secured, covering the whole light curve. The scatter of the observations of 0 mo2 in B and V. derived from the two comparison stars of different colour index, and which are cluster standards, is quite large for photoelectric work of this kind. It is mainly caused by background troubles of subthreshold- and neighbouring stars of the crowded cluster region. The distance of the variable from the cluster center is about 10 minutes of arc. All observations were reduced for outside the atmosphere and transformed to the UBV system.

\section{Discussion:}

The mean magnitude in maximum light of $\mathrm{V} 78$ is $\overline{\mathrm{V}}=14 \mathrm{~m} 10$, and mean colours are $\overline{(B-V)}=0 m 26$, resp. $\overline{(U-B)}=0$ m.27. My previously given photographic data were $\bar{V}=14 \mathrm{~m} 13$, $\overline{(\mathrm{B}-\mathrm{V})}=\mathrm{om} 21$. Therefore the position of the star in the $\mathrm{c}-\mathrm{m}$ diagram is actually coinciding with the brighter domain of the RRc Lyrae stars of the cluster (GEYER, 1970).

As it can be seen from the two colour diagram of the cluster, V78 shows an UV-deficiency, which is well known from globular cluster stars in this colour range. This is also a very strong argument for the cluster membership of this eclipsing binary.

The Figure shows the $\mathrm{V}$ and $\mathrm{B}$ light curves. The different symbols indicate different observing nights; each is the mean of two successive individual observations. The following conclusions can be drawn from the light curve:

1) Little or no disturbances occur in the primary minimum, and which appears quite symmetrical. The object is redder during this phase. This indicates that the smaller and hotter component is eclipsed.

2) Outside the primary minimum large disturbances occur which amount up to om 5 , and with change rapidly in their position within the light curve. 


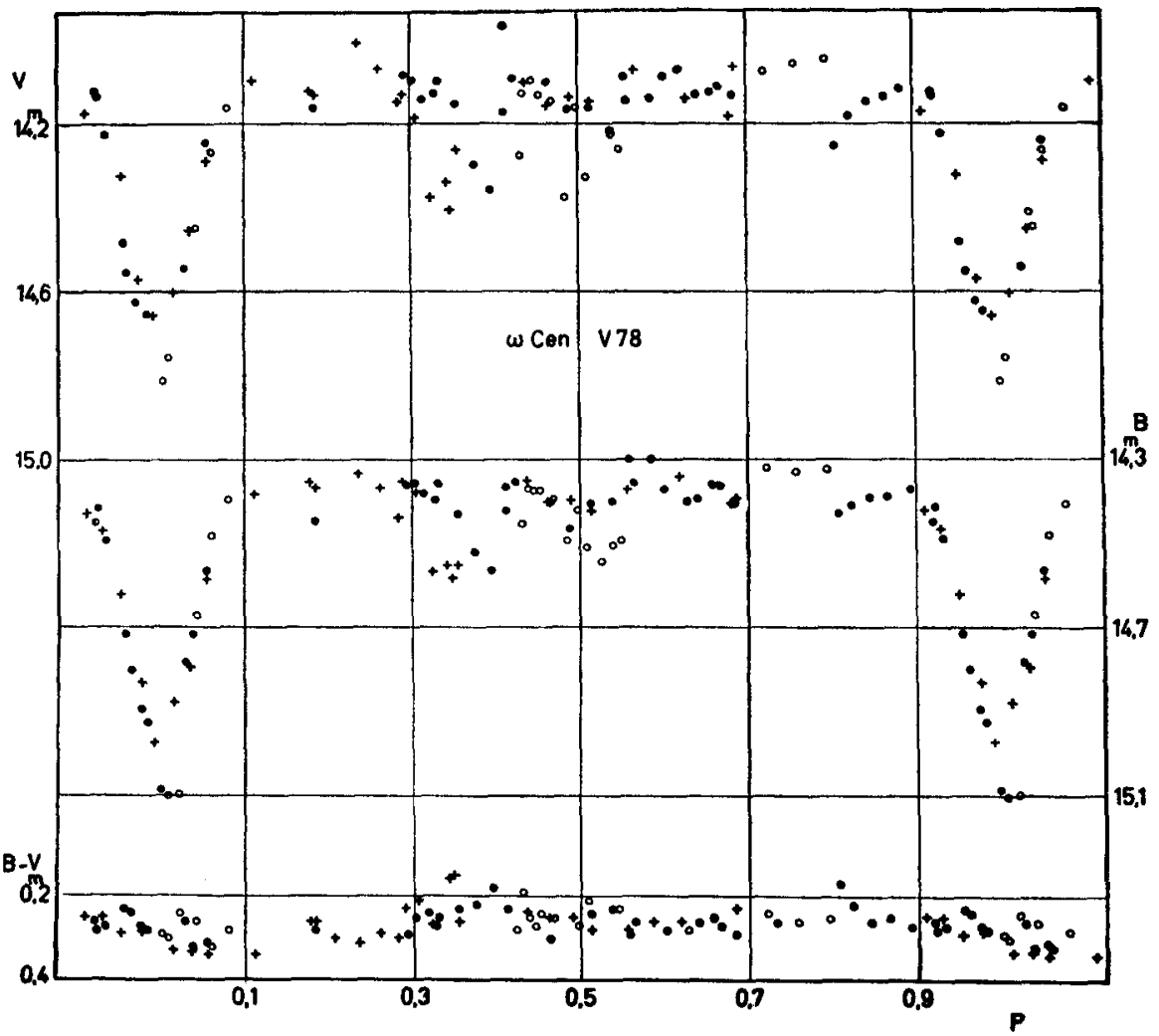

$\mathrm{V}$ and $\mathrm{B}$ light curves of V 78 in Omega Centauri.

3) There exists a very shallow secondary minimum, sometimes hidden by these light fluctuations. The colour index is smaller than the primary minimum.

4) The overall feature of the light curve is of an Algol type.

From these and the above mentioned facts I come to the conclusion that one component - the smaller and hotter one - is an intrinsic variable, and I am inclined to see in it a $R R c$ Lyrae type variable.

Disregarding the mentioned disturbances of the light curve, orbital elements were derived of the non-rectified curves using SCHNELLER's method. For the primary minimum the solution is "occultation", as we already suspected from the behaviour of the colour index.

Though we do not have spectroscopic and radial velocity observations, we may obtain further rough informations about the dimensions of the components by the following considerations: Generally the turn-off point from the main-sequence in the HR diagram of globular clusters is about 3 to 3,5 magnitudes fainter than the horizontal branch. From the data of the table we can therefore see that the larger and less luminous component should be a $G$ type subgiant of luminosity class IV to III, and the horizontal branch star component (RRc Lyrae type variable?) has only a slightly smaller radius than this $G$ subgiant. 
Table: Elements of V 78 in NGC 5139

\begin{tabular}{|c|c|c|}
\hline & V light curve & B light curve \\
\hline Max. brightness & $14 \mathrm{~m} 10$ & $14 \mathrm{~m} 36$ \\
\hline Prim. min. brightness & 14.82 & 15.12 \\
\hline Sec. min. brightness & 14.165: & $14.425:$ \\
\hline Limb dark. coefficient & 0.6 (adopted) & 0.8 (adopted) \\
\hline k & 0.79 & 0.80 \\
\hline$\alpha_{0}^{\text {oc }}$ & 0.85 & 0.85 \\
\hline$r_{g}$ & 0.302 & 0.323 \\
\hline $\begin{array}{l}\mathrm{r}_{\mathrm{s}} \\
\mathrm{i}\end{array}$ & $\begin{array}{c}0.239 \\
79 \% 9\end{array}$ & $\begin{array}{c}0.248 \\
790_{5}\end{array}$ \\
\hline $\mathrm{J}_{8} / \mathrm{J}_{\mathrm{g}}$ & 8.35 : & 8.67 : \\
\hline $\mathrm{Lg}_{\mathrm{g}}$ & $0.16:(1 \mathrm{mgs})$ & $0.15(2 \mathrm{mo4}:)$ \\
\hline $\mathrm{L}_{\mathrm{s}}$ & 0.84 (om19) & $0.85\left(0^{m} 18\right)$ \\
\hline magnitude $m_{g}$ & 16m06: & $16 \mathrm{~m} 38:$ \\
\hline magnitude $\mathrm{m}_{\mathrm{s}}$ & $14 \mathrm{~m} 27$ & $14 \mathrm{~m} 52$ \\
\hline
\end{tabular}

Ref e r e n ces :

BAILEY, S. I., 1902, Harvard Ann. 38.

DICKENS, R. J., and SAUNDERS, J., 1965, Roy. Observ. Bull. 101.

GEYER, E. H., 1967, Z. Astrophys. 66, 16.

GEYER, E. H., and SZEIDL, B. 1970, Astron. Astrophys. 4, 40.

MARTIN, W. Chr., 1938, Ann. Leiden XXII, Nr. 2.

SCHNELLER, H., 1949, Veröff. Sonneberg 1, 359 .

SISTERO, R. F., 1968, I.A.U. Inf. Bull. Var. Stars No. 316

SISTERO, R. F,, C. R. FOURCADE and J. R. LABORDE, 1969, I.A.U. Inf. Bull. Var. Stars No. 402.

\section{Discussion to the paper of GEYER}

HALL: If you plot the colours of the hot star alone, rather than the colour of both stars combined, in the two colour diagram, the position will move up and to the left in such a way as to decrease and perhaps to remove the ultraviolet deficiency which you noted.

GEYER : Yes, a slight reduction of the UV deficiency is possible.

$\mathrm{KOCH}$ : If your interpretation is correct, this is prospectively a binary which has twice independently gone through what theoreticians call Case A of mass exchange.

GEYER : Yes, you may be right.

$\mathrm{KOCH}$ : My second comment is that, within the scatter of the observations, it is theoretically possible for the period to be doubled. What I meant to imply is that the eclipses could each be $0 m 75$ deep, which is theoretically possible.

GEYER: From the observations I do not see the possibility to double the period. 